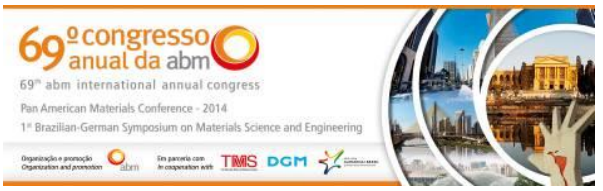

Tema: Metalurgia física e comportamento de materiais em temperaturas elevadas

\title{
AVALIAÇÃO MICROESTRUTURAL DE LIGAS Fe-Ni-Cr SUBMETIDAS À CORROSÃO EM ALTAS TEMPERATURAS*
}

\author{
Maurício de Jesus Monteiro ${ }^{1}$ \\ Sheyla Santana de Carvalho² \\ Cássio Barbosa ${ }^{3}$ \\ lêda Maria Vieira Caminha ${ }^{3}$ \\ Ibrahim de Cerqueira Abud ${ }^{4}$ \\ Claudio Teodoro dos Santos ${ }^{3}$ \\ Paulo Moura Bispo de Santana ${ }^{5}$
}

\section{Resumo}

O presente estudo apresenta a avaliação microestrutural de duas ligas $\mathrm{Fe}-\mathrm{Ni}-\mathrm{Cr}$ com composições químicas distintas submetidas à corrosão em altas temperaturas em tubos de pirólise em uma planta de fabricação de polímeros durante três anos. A superfície e a seção transversal das ligas foram caracterizadas por microscopia eletrônica de varredura e espectroscopia por dispersão de energia de Raios-X. As condições de serviço induziram a formação de uma camada de óxido na superfície das ligas composta por uma região externa e outra interna além de uma região empobrecida em cromo abaixo da camada de óxido. Uma das ligas apresentou também uma zona de oxidação interna. O estágio de modificação microestrutural de ambas as ligas foi analisado e comparado com resultados de outros autores.

Palavras-chave: Ligas Fe-Ni-Cr; Alta temperatura; Tubo de pirólise; Corrosão.

\section{MICROSTRUCTURAL EVALUATION OF Fe-Ni-Cr ALLOYS SUBJECT TO HIGH TEMPERATURE CORROSION}

\section{Abstract}

This study presents the microstructural evaluation of two Fe-Ni-Cr alloys with different chemical compositions used for three years as furnace tubes in pyrolysis process. The alloys were characterized by scanning electron microscopy and energy dispersive X-ray spectroscopy. The service conditions induced the formation of an oxide layer composed by two different regions and a chromium depleted region beneath the oxide layer. One of the alloys also presented an internal oxidation zone. The stage of microstructural degradation of both alloys was analyzed and compared with results of other studies.

Keywords: Fe-Ni-Cr alloys; High temperature; Pirolysis tubes; Corrosion.

1 Engenheiro Mecânico, D.Sc., Tecnologista, DEMP, LACPM, Instituto Nacional de Tecnologia (INT), Rio de Janeiro, RJ, Brasil.

2 Engenheira Metalúrgica, M.Sc., Bolsista, DEMP, LACPM, INT, Rio de Janeiro, RJ, Brasil.

3 Engenheiro(a) Metalúrgico(a), D.Sc. Tecnologista, DEMP, LACPM, INT, Rio de Janeiro, RJ, Brasil. 4 Engenheiro Metalúrgico, M.Sc., Tecnologista, DEMP, LACPM, INT, Rio de Janeiro, RJ, Brasil.

5 Engenheiro Mecânico, M.Sc., Professor, DEM, Instituto Federal da Bahia (IFBA), Salvador, BA, Brasil.

* Contribuição técnica ao $69^{\circ}$ Congresso Anual da ABM - Internacional e ao 14ํㅡㄹ ENEMET - Encontro Nacional de Estudantes de Engenharia Metalúrgica, de Materiais e de Minas, 21 a 25 de julho de 2014, São Paulo, SP, Brasil. 


\section{INTRODUÇÃO}

Ligas Fe-Ni-Cr são frequentemente utilizadas em reatores industriais que trabalham em condições de temperatura elevada e em atmosfera gasosa com alto teor de carbono. Esse ambiente extremamente agressivo é característico de sistemas de conversão do carvão e de diversos processos químicos e petroquímicos. Durante o processo de pirólise uma mistura de hidrocarbonetos e vapor de água passa por uma tubulação que é aquecida externamente por queimadores. Desta forma, o material dos tubos fica exposto tanto ao processo de oxidação quanto ao de carburização [1-3].

A taxa de reação dos metais com a atmosfera gasosa aumenta rapidamente com o aumento da temperatura, o que acarreta em um desgaste severo desses materiais quando expostos a altas temperaturas. Desta forma, a resistência à oxidação é uma característica de fundamental importância para aplicação de ligas metálicas em condições de altas temperaturas [4,5].

O fenômeno de carburização ocorre em temperaturas superiores a $900^{\circ} \mathrm{C}$ sendo caracterizado pela formação de carbonetos devido à difusão do carbono da atmosfera gasosa para o interior do material. $O$ aumento de volume promovido pela formação de carbonetos gera tensões no interior do material. Além disso, a carburização promove a deterioração da ductilidade e da tenacidade dos materiais em baixa temperatura. Assim, trincas podem ser geradas durante a condição de serviço e falhas do material podem ocorrer durante o processo de resfriamento [6].

A carburização pode ser efetivamente evitada pela formação de uma camada contínua e aderente de cromia na superfície da liga, que não permite uma difusão considerável de carbono em direção ao interior da liga. Desta forma, ligas formadoras de $\mathrm{Cr}_{2} \mathrm{O}_{3}$, tais como as do sistema Fe-Cr-Ni, podem permanecer em serviço durante anos sem apresentar danos causados pelo fenômeno de carburização em temperaturas de até $1050^{\circ} \mathrm{C}[1,6]$.

A deposição de carbono na parede interna de tubos de pirólise diminui a transferência térmica, requisitando um procedimento operacional de aumento da temperatura de serviço, para que a eficiência do processo seja mantida. Desta forma, temperaturas superiores a $1050^{\circ} \mathrm{C}$ podem ser alcançadas. Nessas temperaturas, a camada protetora de cromia pode sofrer falhas devido à conversão do óxido de cromo em carbonetos [6-8], expondo a liga à atmosfera carburizante.

O presente estudo tem como objetivo realizar uma avaliação microestrutural de duas ligas $\mathrm{Fe}-\mathrm{Cr}-\mathrm{Ni}$ após serem utilizadas em tubos de pirólises em uma planta de fabricação de polímeros, caracterizando tanto a matriz quanto a camada de óxido formada na superfície das ligas. O estágio de modificação microestrutural das ligas é analisado e discutido.

\section{MATERIAIS E MÉTODOS}

Amostras foram retiradas de um tubo de pirólise após três anos de serviço em uma planta de fabricação de polímeros. Durante esse período, o tubo foi exposto a gases típicos do processo de pirólise $\left(\mathrm{CH}_{4}+\mathrm{H}_{2} \mathrm{O}=\mathrm{CO}+3 \mathrm{H}_{2}\right)$ em uma faixa de temperatura de $850^{\circ} \mathrm{C}$ a $1100^{\circ} \mathrm{C}$. O tubo foi fabricado com duas ligas Fe-Cr-Ni de tal forma que as liga foram submetida a temperaturas diferentes.

Depois do procedimento para o corte do tubo, uma máquina de corte de precisão foi utilizada para a retirada de amostras com aproximadamente $20 \mathrm{~mm}$ de comprimento dos tubos que possuíam cerca de $7 \mathrm{~mm}$ de espessura.

\footnotetext{
* Contribuição técnica ao 69 Congresso Anual da ABM - Internacional e ao 14ํㅡㄹ ENEMET - Encontro Nacional de Estudantes de Engenharia Metalúrgica, de Materiais e de Minas, 21 a 25 de julho de 2014, São Paulo, SP, Brasil.
} 


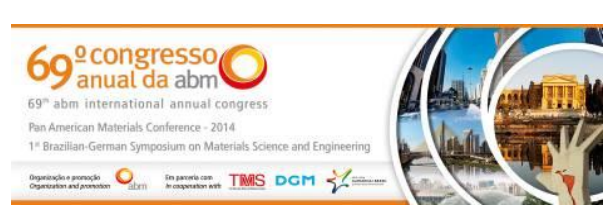

A superfície interna dos tubos foi analisada por Microscopia Eletrônica de Varredura (MEV) e por Espectroscopia por Dispersão de energia de Raios-X (EDX) para caracterização tanto do produto de corrosão quanto do depósito dos elementos químicos característicos do processo de pirólise.

Com o objetivo de analisar a seção transversal por MEV e EDX, as amostras foram embutidas a quente em uma resina condutora, lixadas em papel de SiC até 600 mesh e polidas com pasta de diamante até $3 \mu \mathrm{m}$.

\section{RESULTADOS E DISCUSSÃO}

A média de três análises de EDX realizadas em regiões distintas do centro das amostras das duas ligas, que de agora em diante serão chamadas de amostra $A$ e amostra $B$, é apresentada na tabela 1. Esses resultados sugerem que as amostras $A$ e B sejam respectivamente as ligas ET45Micro e G4852Micro. A amostra A possui maiores teores de cromo e níquel e menor teor de ferro quando comparada com a amostra B. Além dos elementos citados, destaca-se a identificação dos elementos silício e nióbio em ambas as amostras.

Tabela 1. Composição química das ligas. Resultado da média de três análises de EDX (\% em peso)

\begin{tabular}{|c|c|c|c|c|c|c|c|}
\hline Amostra & $\mathrm{C}$ & $\mathrm{Cr}$ & $\mathrm{Fe}$ & $\mathrm{Ni}$ & $\mathrm{Si}$ & $\mathrm{Nb}$ & $\mathrm{Mn}$ \\
\hline $\mathrm{A}$ & 3,27 & 36,82 & 13,87 & 42,83 & 2,15 & 1,07 & - \\
\hline $\mathrm{B}$ & 3,43 & 26,26 & 34,10 & 31,37 & 1,81 & 1,17 & 1,34 \\
\hline
\end{tabular}

As imagens de MEV obtidas em regiões próximas às superfícies internas das amostras A e B (figuras 1 e 2) com contraste de elétrons retroespalhados (BSE) e os resultados das análises de EDX apresentados nas tabelas 2 e 3 revelam que as microestruturas de ambas as amostras são compostas majoritariamente por uma fase rica em cromo (cinza escuro) e outra rica em níquel e ferro (cinza claro), partículas claras ricas em nióbio também são observadas.

Voicu et al. [9] caracterizaram ligas comerciais HP40Nb antes e após tratamentos térmicos realizados a $1000^{\circ} \mathrm{C}$ demonstrando o efeito do envelhecimento na evolução microestrutural dessas ligas. Outros pesquisadores [3,10] caracterizaram modificações ocorridas na microestrutura da liga HK40 após exposição prolongada em atmosferas típicas de oxidação/carburização a $800^{\circ} \mathrm{C}$. No presente estudo, devido ao longo tempo de exposição ao ambiente típico do processo de pirólise ao qual as ligas foram submetidas alterações microestruturais ocorreram, fazendo com que as microestruturas apresentadas nas figuras 1 e 2 não sejam representativas das microestruturas originais das ligas.

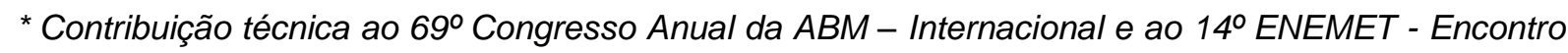
Nacional de Estudantes de Engenharia Metalúrgica, de Materiais e de Minas, 21 a 25 de julho de 2014, São Paulo, SP, Brasil. 


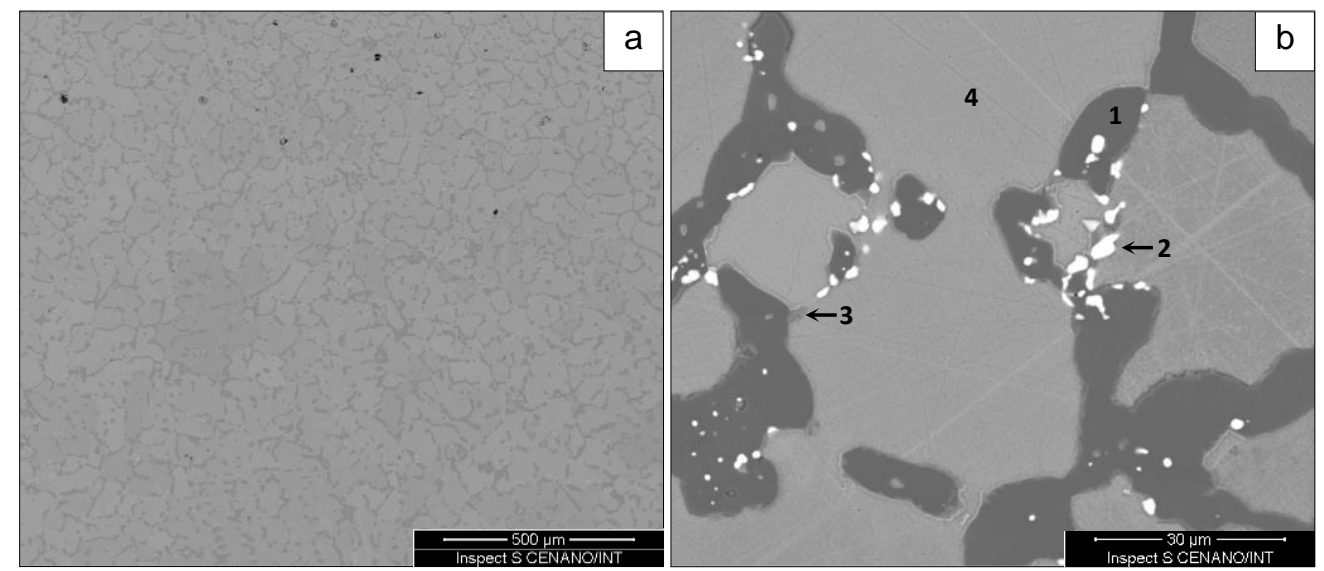

Figura 1. Imagens de MEV - BSE (seção transversal). Região perto da superfície da amostra A. (a) visão geral, (b) visão ampliada, números indicam regiões onde foram realizadas análises de EDX.

Tabela 2. Resultados das análises de EDX das regiões identificadas na figura $1 \mathrm{~b}$ (\% em peso)

\begin{tabular}{|c|c|c|c|c|c|c|c|c|c|}
\hline & $\mathrm{C}$ & $\mathrm{Cr}$ & $\mathrm{Fe}$ & $\mathrm{Ni}$ & $\mathrm{Si}$ & $\mathrm{Mn}$ & $\mathrm{O}$ & $\mathrm{Nb}$ & $\mathrm{Ti}$ \\
\hline 1 & 5,74 & 84,74 & 4,56 & 1,81 & & & 3,13 & & \\
\hline 2 & & 4,02 & 3,98 & 11,87 & & & & 77,93 & 2,20 \\
\hline 3 & 5,23 & 40,49 & 2,49 & 35,35 & 8,66 & & 1,80 & 5,98 & \\
\hline 4 & 4,72 & 10,95 & 17,69 & 62,36 & 2,69 & 1,59 & & & \\
\hline
\end{tabular}

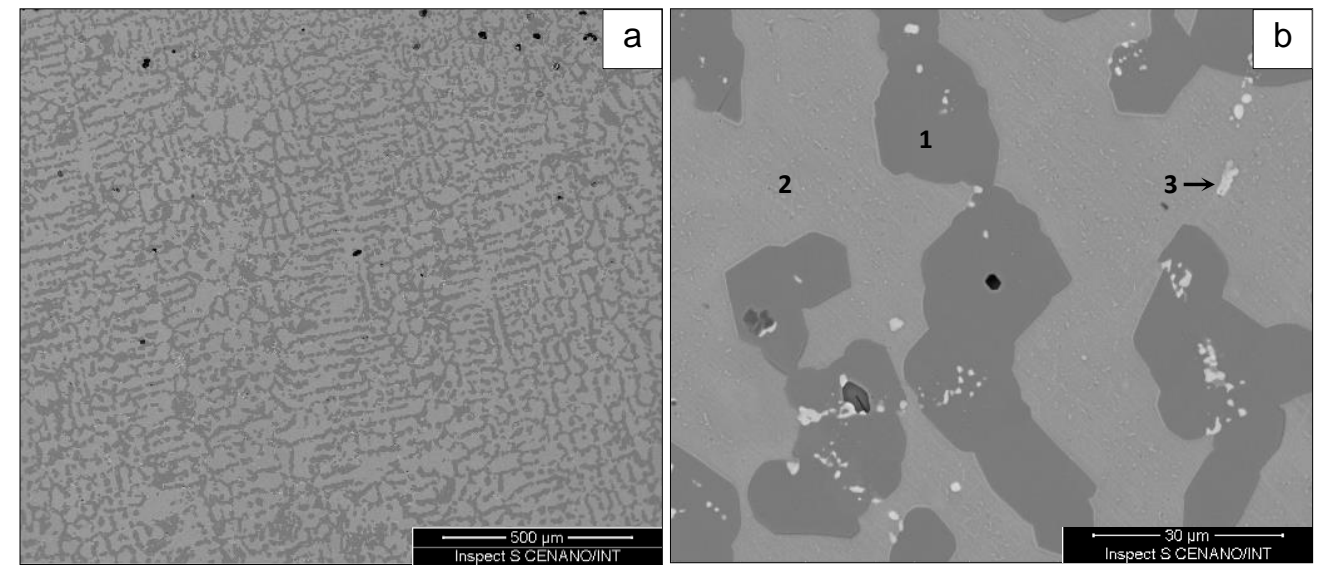

Figura 2. Imagens de MEV - BSE de região perto da superfície da amostra B (seção transversal). (a) visão geral, (b) visão ampliada, números indicam regiões onde foram realizadas análises de EDX.

Tabela 3. Análises de EDX das regiões identificadas na figura $2 b$ (\% em peso)

\begin{tabular}{|c|c|c|c|c|c|c|c|c|}
\hline & $\mathbf{C}$ & $\mathbf{C r}$ & $\mathbf{F e}$ & $\mathbf{N i}$ & $\mathbf{S i}$ & $\mathbf{M n}$ & $\mathbf{N b}$ & $\mathbf{T i}$ \\
\hline 1 & 5,19 & 66,35 & 24,87 & 2,16 & & 1,43 & & \\
\hline 2 & 3,13 & 4,34 & 39,29 & 49,04 & 3,00 & 1,20 & & \\
\hline 3 & 5,34 & 4,24 & 9,61 & & 0,94 & 0,51 & 73,74 & 5,63 \\
\hline
\end{tabular}

Foram observados depósitos de elementos, principalmente carbono, característicos do processo de pirólise nas superfícies das amostras, conforme mostram as imagens de MEV com contraste de elétrons secundários (SE) e as análises de EDX (figura 3 e tabelas 4 e 5 , respectivamente). As irregularidades de altura observadas na figura 3 são consequências tanto do processo industrial quanto do corte do tubo, que podem ter sido responsáveis pelo desprendimento de certas regiões do depósito. Alguns elementos típicos das ligas também foram identificados.

\footnotetext{
* Contribuição técnica ao $69^{\circ}$ Congresso Anual da ABM - Internacional e ao 14ํㅡㄹ ENEMET - Encontro Nacional de Estudantes de Engenharia Metalúrgica, de Materiais e de Minas, 21 a 25 de julho de 2014, São Paulo, SP, Brasil.
} 
Figura 4. Imagens de MEV - BSE da seção transversal da amostra A. (a) visão geral, (b) visão ampliada.

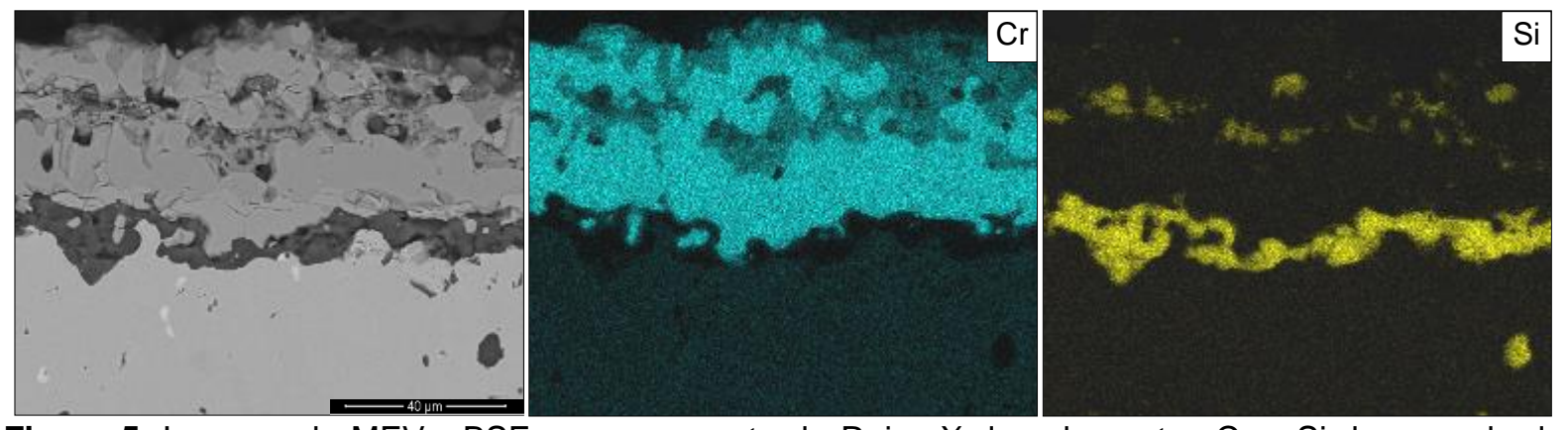

Figura 5. Imagem de MEV - BSE e mapeamento de Raios-X dos elementos $\mathrm{Cr}$ e Si da camada de óxido formada na amostra A (seção transversal).
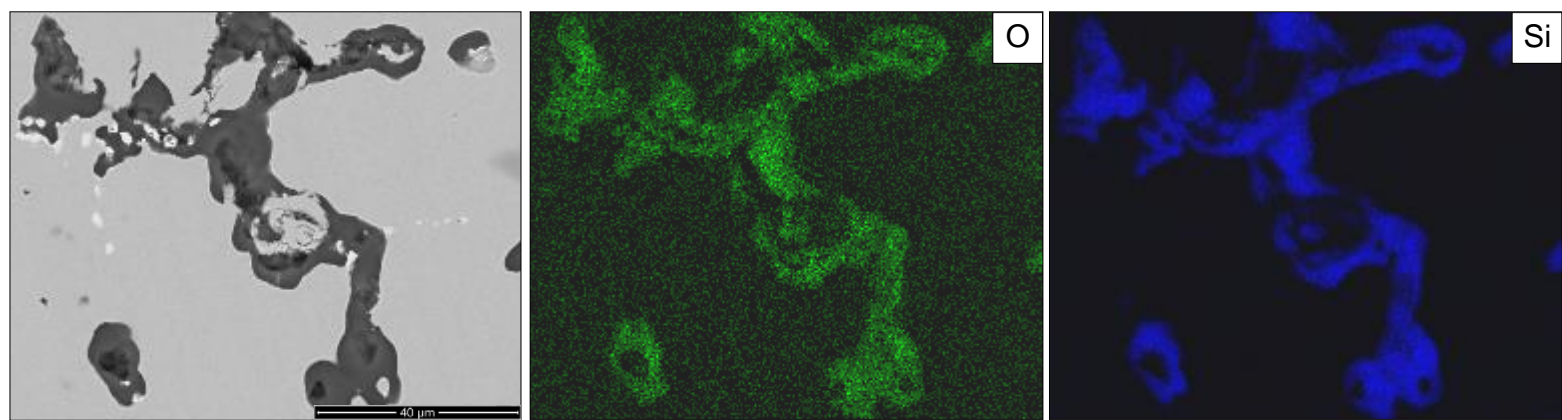

Figura 6. Imagem de MEV - BSE e mapeamento de Raios-X dos elementos O e Si da zona de oxidação interna da amostra A (seção transversal).

A formação de uma camada de óxido composta por uma região externa rica em cromo e uma região interna rica em silício e a caracterização de uma zona de oxidação interna formada por óxido rico em silício na superfície da amostra $A$ também são evidenciadas na figura 7. Além disso, essa figura mostra o depósito de carbono, proveniente do processo de pirólise, praticamente contínuo em toda a extensão da superfície acima da camada de óxido e a distribuição dos elementos $\mathrm{O}$, $\mathrm{Nb}, \mathrm{Ni}$ e Fe na liga. É possível observar que existe uma região, de cerca de $250 \mu \mathrm{m}$, abaixo da camada de óxido que não apresenta a fase rica em Cr. Vale ressaltar que os elementos $\mathrm{Ni}$ e $\mathrm{Fe}$ estão restritos à liga, ou seja, não constituem a camada de óxido, e que o elemento $\mathrm{Nb}$ está distribuído por toda liga, inclusive na região que não apresenta a fase rica em $\mathrm{Cr}$.

* Contribuição técnica ao 69 Congresso Anual da ABM - Internacional e ao 14ํㅡㄹ ENET - Encontro Nacional de Estudantes de Engenharia Metalúrgica, de Materiais e de Minas, 21 a 25 de julho de 2014, São Paulo, SP, Brasil. 

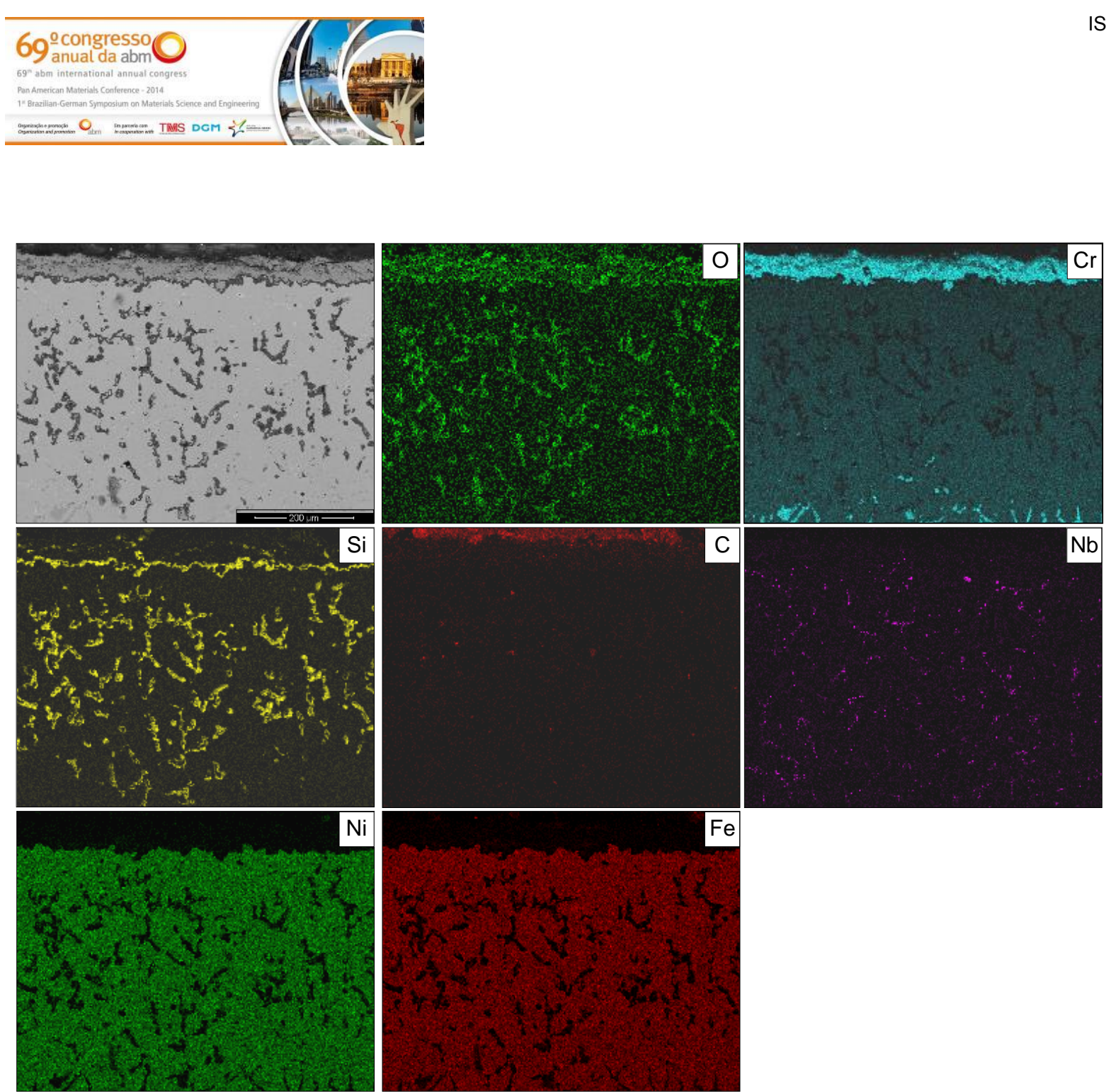

Figura 7. Imagem de MEV - BSE e mapeamento de Raios-X dos elementos O, Cr, Si, C, Nb, Ni e Fe da amostra A (seção transversal).

As figuras $8 \mathrm{a}$ e $8 \mathrm{~b}$ apresentam imagens de MEV com contraste de BSE da seção transversal da amostra B.
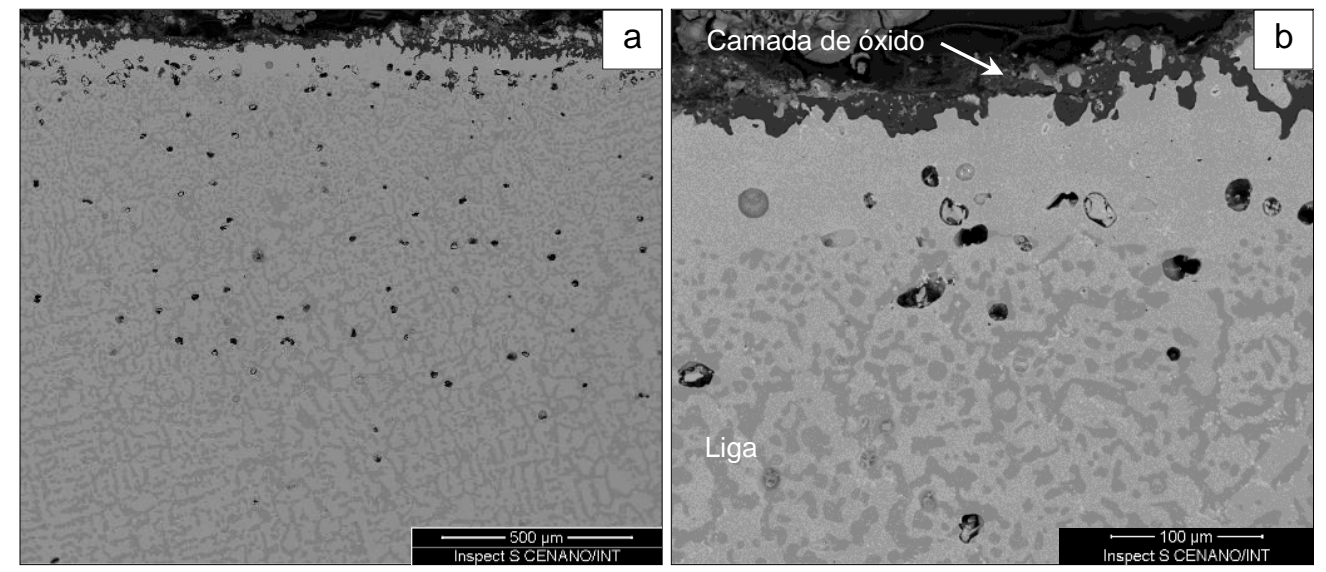

Figura 8. Imagens de MEV - BSE da seção transversal da amostra B. (a) visão geral, (b) visão ampliada.

Foi evidenciada a formação de uma fina e irregular camada de óxido na superfície da amostra B (aproximadamente $15 \mu \mathrm{m}$ ), além de uma região empobrecida em $\mathrm{Cr}$

* Contribuição técnica ao 69ํㅡㄹ Congresso Anual da ABM - Internacional e ao 14º ENEMET - Encontro Nacional de Estudantes de Engenharia Metalúrgica, de Materiais e de Minas, 21 a 25 de julho de 2014, São Paulo, SP, Brasil. 
abaixo dessa camada, com espessura de aproximadamente $90 \mu \mathrm{m}$, conforme mostram as figuras $8 \mathrm{a}$ e $8 \mathrm{~b}$.

A exemplo da amostra $A$, a camada de óxido formada na superfície da amostra $B$ é composta por uma região externa rica em cromo e outra interna rica em silício, provavelmente $\mathrm{Cr}_{2} \mathrm{O}_{3}$ e $\mathrm{SiO}_{2}$ respectivamente (figuras 9 e 10).
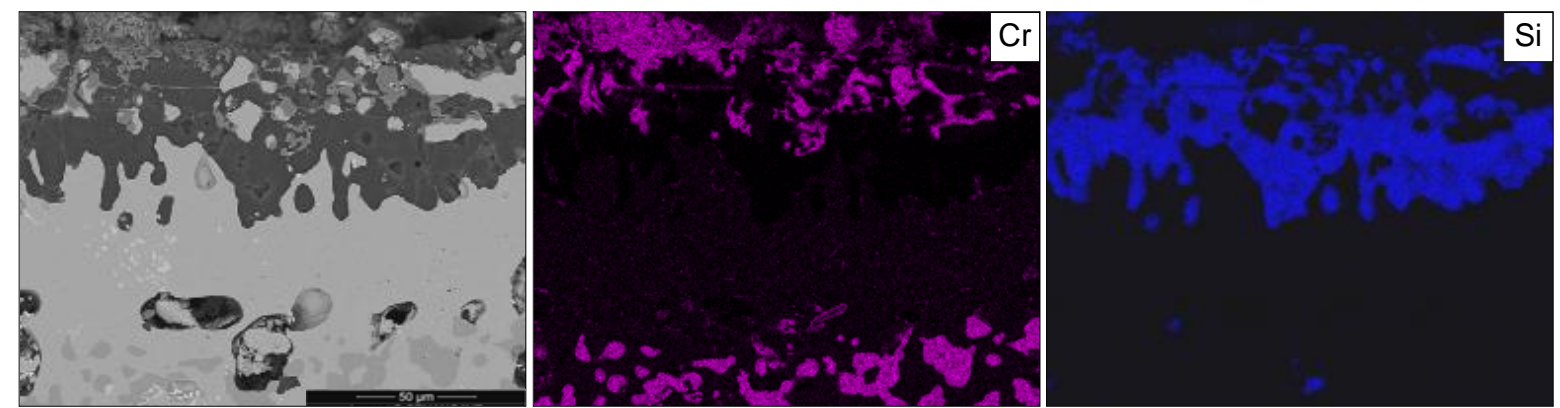

Figura 9. Imagem de MEV - BSE e mapeamento de Raios-X dos elementos $\mathrm{Cr}$ e Si da camada de óxido formada na amostra B (seção transversal).

A pequena espessura da camada de óxido está relacionada com o desprendimento da região externa de óxido rico em cromo, que pode ter ocorrido durante os processos de resfriamento do tubo ou de preparação da amostra. O desprendimento dessa região é evidenciado pela ausência de uma camada de carbono contínua acima da camada de óxido, conforme mostra o mapeamento do elemento carbono na figura 10. Pela distribuição dos elementos $\mathrm{O}, \mathrm{Cr}$, Nb, Ni e Fe é possível observar que, assim como na amostra $\mathrm{A}$, na amostra $\mathrm{B}$ os elementos $\mathrm{Ni}$ e $\mathrm{Fe}$ estão restritos à liga e que o elemento $\mathrm{Nb}$ está distribuído por toda liga, inclusive na região empobrecida em Cr.

* Contribuição técnica ao 69 Congresso Anual da ABM - Internacional e ao 14ํㅡN ENET - Encontro Nacional de Estudantes de Engenharia Metalúrgica, de Materiais e de Minas, 21 a 25 de julho de 2014, São Paulo, SP, Brasil. 

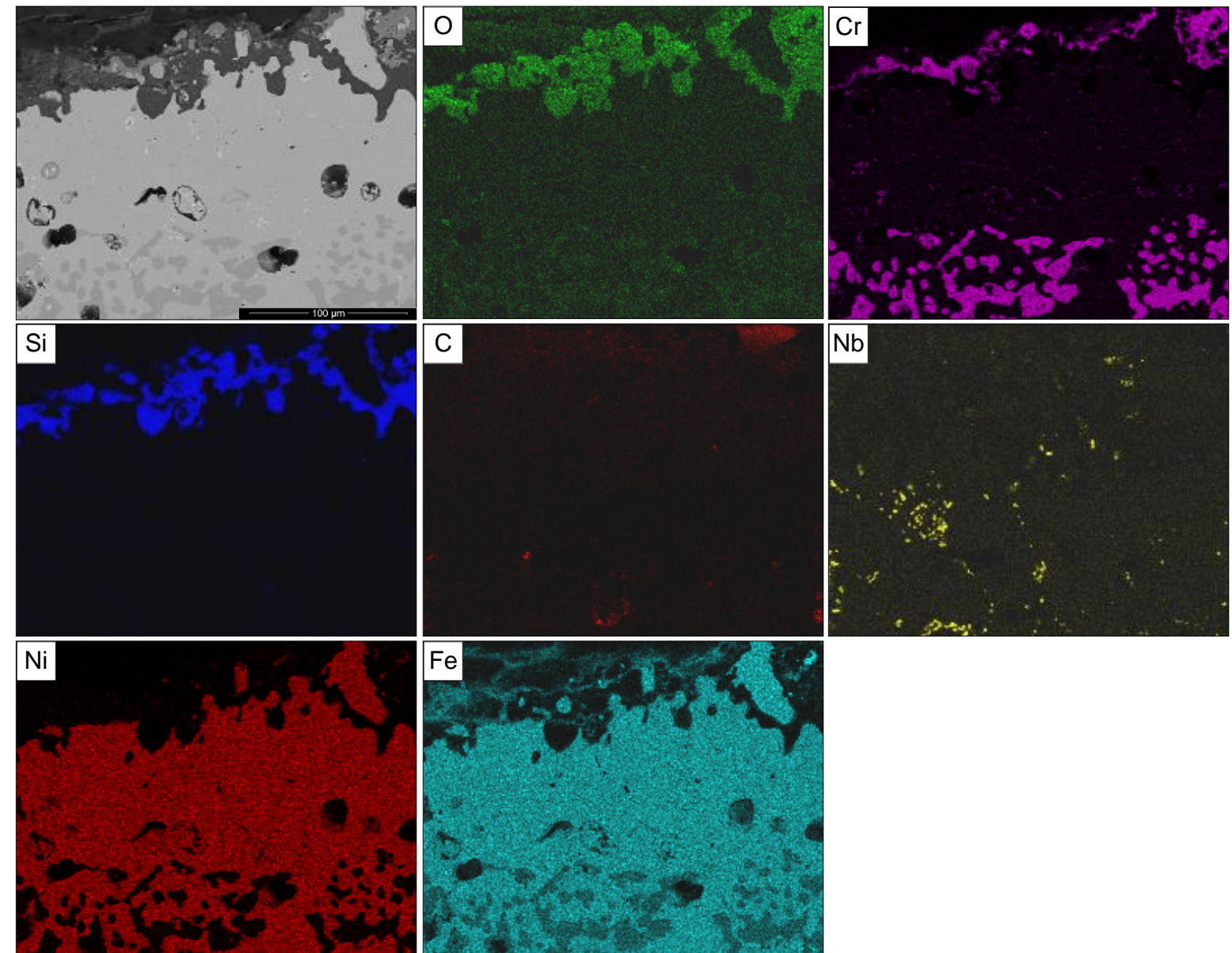

Figura 10. Imagem de MEV - BSE e mapeamento de Raios-X dos elementos $\mathrm{O}, \mathrm{Cr}, \mathrm{Si}, \mathrm{C}, \mathrm{Nb}, \mathrm{Ni}$ e $\mathrm{Fe}$ da amostra B (seção transversal).

O estudo realizado por Petkovic-Luton e Ramanarayanan [11] sobre oxidação de ligas $\mathrm{Fe}-\mathrm{Ni}-\mathrm{Cr}$ em atmosferas contendo oxigênio e carbono resultou na formulação de um modelo que propõe que o material seja submetido aos seguintes estágios de modificação microestrutural durante a sua degradação:

I. Formação inicial de uma camada de óxido;

II. Oxidação em atmosfera contendo carbono;

III. Redistribuição interna dos elementos cromo e carbono;

IV. Difusão de carbono em direção à liga;

V. Oxidação dos carbonetos.

Outros estudos $[3,10,12,13]$, de um modo geral, validaram esse modelo, sendo que algumas alterações dependendo da composição química da liga e das temperaturas estudadas foram sugeridas. Além disso, tentativas de modelagem computacional do processo de carburização foram realizadas.

Os três estágios iniciais desse modelo microestrutural geralmente constituem o maior tempo de vida útil do material. Em linhas gerais, o estágio I é caracterizado pela formação de uma camada de óxido composta por $\mathrm{Cr}_{2} \mathrm{O}_{3}$ e $(\mathrm{Fe}, \mathrm{Ni}, \mathrm{Cr})_{\times} \mathrm{O}_{y}$ e os estágios II e III são caracterizados pela formação de $\mathrm{Cr}_{2} \mathrm{O}_{3}$ e $\mathrm{SiO}_{2}$ ou $\mathrm{Al}_{2} \mathrm{O}_{3}$ além de uma região empobrecida em cromo abaixo da camada de óxido. Nos estágios IV e V não há formação de uma camada protetora de cromia devido ao empobrecimento do teor de $\mathrm{Cr}$ até um valor abaixo do crítico (10\% em peso) na região adjacente à superfície da liga $[11,12]$.

* Contribuição técnica ao 69 Congresso Anual da ABM - Internacional e ao 14ํㅡㄹ ENET - Encontro Nacional de Estudantes de Engenharia Metalúrgica, de Materiais e de Minas, 21 a 25 de julho de 2014, São Paulo, SP, Brasil. 


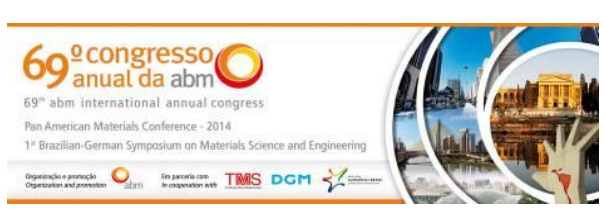

Os resultados apresentados no presente trabalho mostram que a camada de óxido formada nas superfícies das amostras A e B é composta por uma região externa rica em cromo e por uma região interna rica em silício (mapeamentos dos elementos $\mathrm{Cr}$, Si e O das figuras 5, 6, 7, 9 e 10) com a formação de uma região empobrecida em Cr abaixo da camada de óxido conforme mostram as figuras 4 e 8 e os mapeamentos do elemento $\mathrm{Cr}$ nas figuras 7 e 10. Além disso, não foi identificada a presença de espinélio ( $\mathrm{Fe}, \mathrm{Ni}, \mathrm{Cr}$ ) $\mathrm{O}$ y na camada de óxido conforme mostram os mapeamentos dos elementos $\mathrm{Fe}$, $\mathrm{Ni}$ e $\mathrm{O}$ das figuras 7 e 10. Estes resultados validam o modelo proposto por Petkovic-Luton e Ramanarayanan [11], pois se enquadram nas características dos estágios de modificação microestrutural II e III. A existência de uma região composta por óxido de cromo e a não identificação de óxido complexo (espinélio) na camada de óxido indicam, respectivamente, que o estágio IV não foi alcançado e que o tempo de serviço foi suficiente para que o estágio I fosse finalizado. Os estágios II e III são apresentados esquematicamente na figura 11.

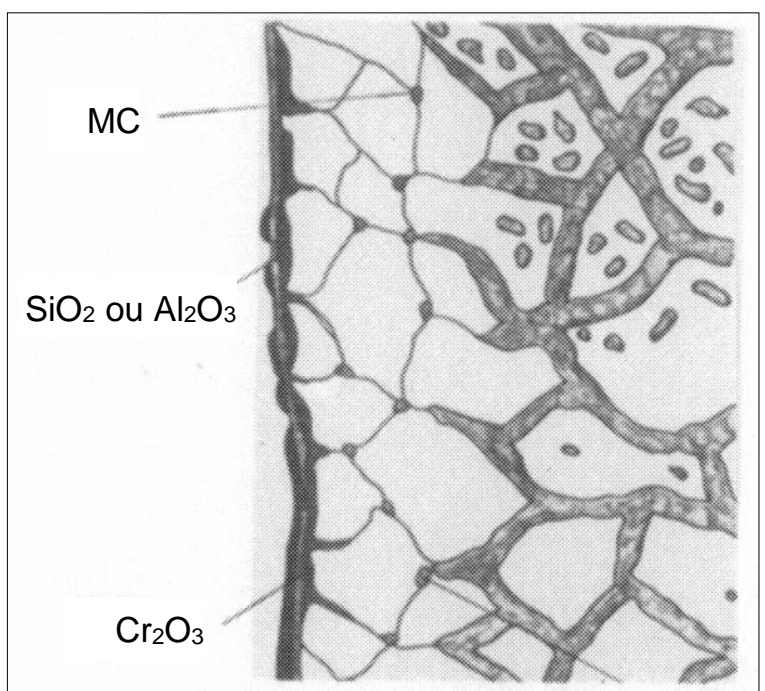

Figura 11. Representação esquemática da variação microestrurural dos estágios II e III proposta no modelo de Petkovic-Luton e Ramanarayanan. Adaptado [11].

Os resultados de mapeamento do elemento $\mathrm{Nb}$ apresentados nas figuras 7 e 10 e o esquema do modelo microestrutural apresentado na figura 11 indicam a existência de carbonetos $\mathrm{NbC}$ em ambas as ligas. Esses carbonetos possuem uma estabilidade termodinâmica muito alta [14,15], continuando estáveis na região empobrecida em Cr.

A formação de uma camada de óxido de cromo envolve a difusão de $\mathrm{Cr}$ da liga em direção à interface óxido / liga. Para manter o crescimento dessa camada, a fase rica em cromo (carboneto de cromo) se dissolve gerando, dessa forma, uma região empobrecida em cromo abaixo da camada de óxido. Uma vez que o carbono não consegue penetrar na camada compacta de óxido de cromo, a presença dessa camada não apenas impede a difusão do carbono da atmosfera em direção à liga mas também força a difusão do carbono proveniente da dissolução da fase rica em cromo em direção à liga, acarretando na mudança de morfologia da fase rica em cromo no interior da liga. A figura 12 mostra esquematicamente esse processo [12]. Dependendo do teor de silício na liga é possível a formação de $\mathrm{SiO}_{2}$, abaixo da camada de cromia, como uma camada contínua e/ou como partículas de oxidação interna [11]. Os teores de ambas as ligas analisadas no presente estudo (tabela 1)

* Contribuição técnica ao 69 Congresso Anual da ABM - Internacional e ao 14ํㅡN ENET - Encontro Nacional de Estudantes de Engenharia Metalúrgica, de Materiais e de Minas, 21 a 25 de julho de 2014, São Paulo, SP, Brasil. 


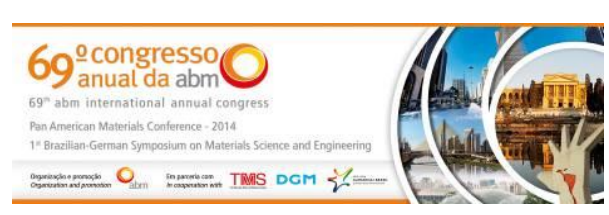

foram suficientes para a formação de uma camada contínua de óxido de silício abaixo da camada de óxido de cromo, conforme mostram os mapeamentos do elemento Si nas figuras 5 e 9 . O teor de silício da amostra A, que é superior ao da amostra $B$, foi suficiente também para permitir a formação de uma região de oxidação interna (mapeamentos dos elementos $\mathrm{O}$ e Si nas figuras 6 e 7).

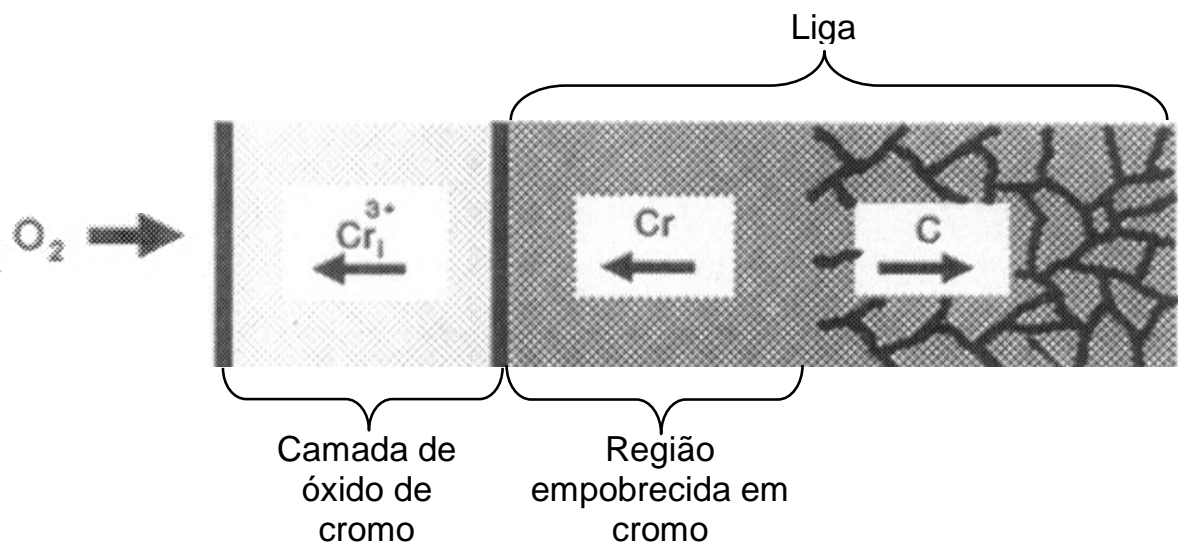

Figura 12. Representação esquemática da variação microestrurural envolvida no crescimento de uma camada de cromo em Fe-Cr-Ni. Adaptado [12].

Embora a amostra A possua maior teor de cromo do que a amostra B (tabela 1), a região empobrecida em cromo caracterizada na amostra $A$ possui uma espessura maior do que a apresentada pela amostra B (figuras $4 \mathrm{~b}$ e 8b), aproximadamente 250 $\mu \mathrm{m}$ e $90 \mu \mathrm{m}$, respectivamente. A espessura dessa região é função do processo difusional na liga, sendo um parâmetro indicativo da resistência da liga ao processo corrosivo. Segundo dados da literatura, existe um valor máximo crítico para a espessura dessa região de $300 \mu \mathrm{m}[11,16]$. Acima desse valor a distância para a difusão do $\mathrm{Cr}$ em direção à superfície da liga se torna um obstáculo muito grande para a continuação do crescimento da camada protetora de óxido de cromo.

Como as amostras foram retiradas de tubos que estavam em serviço, ou seja, não havia controle absoluto das condições nas quais o processo corrosivo ocorreu, a amostra $A$ pode ter sofrido ciclos térmicos mais rigorosos e/ou ter sido exposta à temperatura superior quando comparada com a amostra B.

Ciclos térmicos geram a formação de defeitos estruturais (poros, trincas, etc.) na camada de óxido [17,18], acarretando em uma oxidação interna mais agressiva e em desprendimento da camada de óxido. Sucessivos eventos de desprendimento da camada de óxido geram uma maior necessidade de consumo de $\mathrm{Cr}$ para a regeneração da camada protetora de óxido de cromo, aumentando assim a espessura da região empobrecida nesse elemento na liga.

$\mathrm{O}$ aumento da temperatura acarreta em uma cinética do processo de difusão mais rápida, fazendo com que a região empobrecida em cromo seja mais espessa, principalmente em tempos de exposição mais longos. Além disso, em temperaturas superiores a $1050^{\circ} \mathrm{C}$ a camada protetora de óxido pode sofrer falhas e expor a liga à atmosfera caburizante [6-8]. Estas falhas são resultantes da conversão do óxido de cromo em carbonetos que, por possuírem volume maior do que o óxido, geram trincas na camada. Esse processo de carburização não foi identificado no presente estudo, indicando que a temperatura de serviço dos tubos não deve ter ultrapassado $1050^{\circ} \mathrm{C}$. Desta forma, a carburização não seria a causa da diferença entre as espessuras da região empobrecida em cromo das amostras A e B.

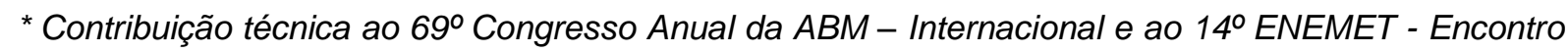
Nacional de Estudantes de Engenharia Metalúrgica, de Materiais e de Minas, 21 a 25 de julho de 2014, São Paulo, SP, Brasil. 


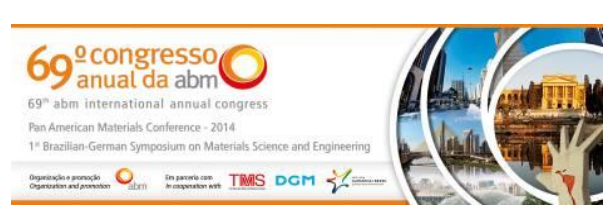

\section{CONCLUSÃO}

O presente estudo de avaliação microestrutural de duas ligas $\mathrm{Fe}-\mathrm{Ni}-\mathrm{Cr}$ com composições químicas distintas, denominadas amostra $A$ e amostra $B$, submetidas à corrosão em altas temperaturas em tubos de pirólise em uma planta de fabricação de polímeros durante três anos permite concluir que:

- A camada de óxido formada em ambas as amostras é composta por uma região externa rica em cromo e uma região interna rica em silício. Não foram formados óxidos complexos (espinélios) contendo ferro, níquel e cromo;

- Houve a formação de uma região empobrecida em cromo em ambas as amostras;

- Ambas as amostras apresentaram características compatíveis com os estágios II e III de modificação microestrutural caracterizados pela redistribuição interna dos elementos cromo e carbono;

- O processo de carburização não foi caracterizado em ambas as amostras;

- A amostra A, com maior teor de silício do que a amostra B, apresentou uma zona de oxidação interna caracterizada pela formação de óxido rico em silício;

- A maior espessura da região empobrecida em cromo apresentada pela amostra A é atribuída a fatores operacionais, tais como exposição a temperaturas superiores e/ou ciclos térmicos mais severos em relação à amostra B.

\section{Agradecimentos}

Os autores expressam seus agradecimentos ao centro de caracterização em nanotecnologia do INT - CENANO - pela utilização dos microscópios eletrônicos, às agências de fomento à pesquisa - FINEP, FAPERJ e CNPq - que apoiam regularmente o CENANO, à PETROBRAS financiadora da compra dos microscópios eletrônicos e a Rafael Vinhosa pelo seu trabalho na preparação das amostras.

\section{REFERÊNCIAS}

1 Forseth S, Kofstad P. Carburization of Fe-Ni-Cr stells in $\mathrm{CH}_{4}-\mathrm{H}_{2}$ mixture at $850-$ $1000^{\circ} \mathrm{C}$. Materials and corrosion, 1998, 49: 266-271.

2 Klöwer J, Heubner U. Carburisation of nickel-base alloys and its effects on the mechanical properties. Materials and corrosion, 1998, 49: 237-245.

3 Kaya AA. Microstructure of HK40 alloy after high-temperature service in oxidizing/carburizing environment II. Carburization and carbide transformations. Materials characterization, 2002, 49: 23-34.

4 Birks N, Meier GH. Introduction to high temperature oxidation of metals, Londres: Edward Arnold, 1982.

5 Kofstad P. High temperature corrosion, Londres: Elsevier Applied Science, 1988.

6 Rahmel A, Grabke HJ, Steinkusch W. Carburization - introductory survey. Materials and corrosion, 1998, 49: 221-225.

7 Bennett MJ, Price JB. A physical and chemical examination of an ethylene steam cracker coke and of the underlying pyrolysis tube. Journal of materials science, 1981, 16: 170-188.

8 Terry BS, Wright J, Hall D. A model for prediction of carburization in steels for ethylene production furnaces. Corrosion Science, 1989, 29, 717 - 734.

9 Voicu R, Andrieu E, Poquillon D, Furtado J, Lacaze J. Microstructure evolution of HP40$\mathrm{Nb}$ alloys during aging under air at $1000^{\circ} \mathrm{C}$. Materials characterization, 2009, 60: 10201027.

* Contribuição técnica ao 69 Congresso Anual da ABM - Internacional e ao 14ํㅡㄹ ENEMET - Encontro Nacional de Estudantes de Engenharia Metalúrgica, de Materiais e de Minas, 21 a 25 de julho de 2014, São Paulo, SP, Brasil. 


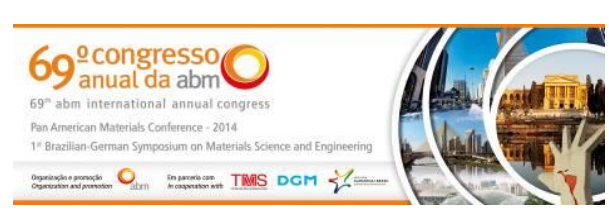

10 Kaya A.A, Krauklis P, Young DJ. Microstructure of HK40 alloy after high-temperature service in oxidizing/carburizing environment I. Oxidation phenomena and propagation of a crack. Materials characterization, 2002, 49: 11-21.

11 Petkovic-Luton R, Ramanarayanan TA. Mixed-Oxidant attack of high-temperature alloys in carbon- and oxygen- containing environments. Oxidation of metals, 1990, 34: 381400.

12 Ling S, Ramanarayanan R, Petkovic-Luton R. Computational modeling of mixed oxidant - carburization process: part 1, Oxidation of Metals, 1993, 40: 179- 196.

13 Brady MP, Gleeson B, Wright IG, Alloy Design Strategies for Promoting Protective Oxide-Scale Formation. Journal of the minerals, metals \& materials society, 2000: 16-21.

14 Farkas DM, Groza JR, Mukherjee AK. Themodynamic analysis of carbides precipitates in a niobium-zirconium-carbon alloy. Scripta Materialia, 1995, 34 (1): 103-110.

15 Grabke HJ. Carburization, carbides formation, metal dusting, coking. Matteriali in technologije, 2002, 36 (6): 297-305.

16 Jakobi D, Gommans R. Typical failures in pyrolysis coils for ethylene cracking. Materials and corrosion, 2003, 54 (11): 881-886.

17 Grabke HJ, Jakobi D. High temperature corrosion of cracking tubes. Materials and corrosion, 2002, 53: 494-499.

18 Rahmel A, Grabke HJ, Steinkusch W. Carburization - introductory survey, Materials and corrosion, 1998, 49: 221-225

* Contribuição técnica ao $69^{\circ}$ Congresso Anual da ABM - Internacional e ao 14ํㅡㄹ ENEMET - Encontro Nacional de Estudantes de Engenharia Metalúrgica, de Materiais e de Minas, 21 a 25 de julho de 2014, São Paulo, SP, Brasil. 